TITLE:

\title{
Spectral and angle-resolved magneto-optical characterization of photonic nanostructures
}

\author{
AUTHORS AND AFFILIATIONS: \\ Mikko Kataja ${ }^{1}$, Rafael Cichelero ${ }^{1,2}$ and Gervasi Herranz ${ }^{1}$ \\ ${ }^{1}$ Institut de Ciència de Materials de Barcelona (ICMAB-CSIC), Campus de la UAB, 08193 \\ Bellaterra, Spain \\ ${ }^{2}$ Department of Physics, University of Gothenburg, Gothenburg 412 96, Sweden
}

\section{KEYWORDS:}

Photonic crystals, magneto-optics, plasmonics, back focal plane measurement, spectroscopy, magnetoplasmonics

\section{SUMMARY:}

Photonic bandstructure enables understanding how confined electromagnetic modes propagate within a photonic crystal. In photonic crystals that incorporate magnetic elements, such confined and resonant optical modes are accompanied by enhanced and modified magneto-optical activity. We describe a measurement procedure to extract the magneto-optical bandstructure by Fourier space microscopy.

\section{ABSTRACT:}

Photonic crystals are periodic nanostructures that can support a variety of confined electromagnetic modes. Such confined modes are usually accompanied by local enhancement of electric field intensity that strengthens light-matter interactions, enabling applications such as Surface-Enhanced Raman Scattering (SERS) and surface plasmon enhanced sensing. In the presence of magneto-optically active materials, the local field enhancement gives rise to anomalous magneto-optical activity. Typically, the confined modes of a given photonic crystal depend strongly on the wavelength and incidence angle of the incident electromagnetic radiation. Thus spectral and angular-resolved measurements are needed to fully identify them as well as to establish their relation with the magneto-optical activity of the crystal. In this article, we describe how to use a Fourier-plane (back focal plane) microscope to characterize magneto-optically active samples. As a model system, we use here a plasmonic grating built out of magneto-optically active $\mathrm{Au} / \mathrm{Co} / \mathrm{Au}$ multilayer. In the experiments we apply a magnetic field on the grating in-situ and measure its reciprocal space response, obtaining the magneto-optical response of the grating over a range of wavelengths and incident angles. This information enables us to build a complete map of the plasmonic bandstructure of the grating and the angle and wavelength dependent magneto-optical activity. These two images allow us to pinpoint the effect that the plasmon resonances have on the magneto-optical response of the grating. The 
relatively small magnitude of magneto-optical effects requires a careful treatment of the acquired optical signals. To this end, an image processing protocol for obtaining magneto-optical response from the acquired raw data is laid out.

\section{INTRODUCTION:}

Confined electromagnetic modes in photonic crystals can arise from a variety of different origins, such as plasmon resonances around metal/dielectric interfaces or Mie resonances in high refractive index dielectric nanostructures ${ }^{1-3}$, and can be designed to appear at specifically defined frequencies ${ }^{4,5}$. Their presence gives rise to many fascinating phenomena such as photonic band gaps ${ }^{6-8}$, strong photon localization ${ }^{9}$, slow light ${ }^{10}$ and Dirac cones ${ }^{11}$. Fourier plane microscopy and spectroscopy are basic tools for characterization of photonic nanostructures as they enable capturing many essential properties of confined modes occurring in them. In Fourier space microscopy, as opposed to conventional real plane imaging, the information is presented as the function of angular coordinates ${ }^{12,13}$. It is alternatively known as back focal plane (BFP) imaging as the angular decomposition of the light emanating from the sample is recorded from the back focal plane of the microscope objective. The angular spectrum, i.e. the far field emission pattern of the sample is related to the momentum of light emanating from it ( $\hbar \mathbf{k})$. In particular, it represents its in-plane momentum $\left(\mathrm{k}_{\mathrm{x}}, \mathrm{k}_{\mathrm{y}}\right)$ distribution ${ }^{14}$.

In magneto-optically active samples, the presence of confined photonic excitations has been shown to result in considerable enhancement of the magneto-optical response ${ }^{15-19}$. Magnetooptical effects depend on the mutual geometry of the magnetic field and the incident electromagnetic radiation. Most commonly encountered magneto-optical geometries for linearly polarized light and their nomenclature are depicted in Figure 1. Here, we demonstrate a setup that can be used to explore two magneto-optical effects that are observed in reflection: transverse and longitudinal magneto-optical Kerr effects, abbreviated, respectively, as TMOKE and LMOKE. TMOKE is an intensity effect, where the reflectivities of the opposing magnetization states are different while LMOKE manifests as a rotation of the reflected light polarization axis. The effects are distinguished by the orientation of the magnetization with respect to the light incidence, where for LMOKE the magnetization is oriented parallel to the in plane component of the wave vector of the light while for TMOKE it is transverse to it. For normally incident light, both in-plane components of the momentum of light are null $\left(k_{x}=k_{y}=0\right)$ and, consequently, both effects are zero. Configurations where both effects are present can be easily conceived. However, to simplify the data analysis, in this demonstration we limit ourselves to situations where only one of the effects is present, namely TMOKE.

Several optical configurations can be used to measure the angular distribution of light emitted from magnetophotonic crystals. E.g. in ${ }^{20}$ and ${ }^{21}$ such a setup was successfully used in transmission geometry to unveil plasmon influence on magneto-optical phenomena. As an illustration, in 22 some possible configurations are presented for a microscope that uses an infinity corrected objective lens. In our configuration, depicted in Figure 2 (A), we use an infinity corrected lens where the light coming from a given point in the sample is directed by the objective lens into collinear beams. In Figure $2(A)$, beams emerging from the top (dashed lines) and the bottom (solid lines) of the sample are schematically depicted. Then, a collecting lens is 
used to refocus these beams to form an image at the image plane (IP). A second lens, also known as Bertrand lens, is then placed after the image plane to separate the incoming light at its focus plane into angular components, depicted in Figure $2(\mathrm{~A})$ in red, blue and black. From this back focal plane, the angular distribution of the light emitted by the sample can be measured with a camera. Effectively, the Bertrand lens performs a Fourier transform on the light beam arriving at it. The spatial intensity distribution at the BFP corresponds to the angular distribution of the incident radiation. A full reciprocal space reflectance map of the sample can be established by illuminating the sample with the same objective that is used to collect the response of the sample. The incoming and out coming beams are separated using a beam splitter. The complete setup is depicted in Figure 3 (A). To obtain a spectrum, a tunable light source or a monochromator is needed. The measurement can then be repeated over different wavelengths, keeping in mind that due to the spectrum of standard light sources, the results need to be normalized to the reflectivity of a control sample. For this purpose, one can use a mirror or a part of the sample that has been purposefully left unpatterned to allow for a high reflectivity. To assist in positioning, we show how to integrate the setup with an additional optical system that enables real-space imaging of the sample, shown in Figure 2 (B).

We now proceed to establish a method for measuring the angular resolved magneto-optical spectrum of a photonic crystal, using as a representative sample a DVD grating covered with an $\mathrm{Au} / \mathrm{Co} / \mathrm{Au}$ film where the presence of ferromagnetic cobalt gives rise to considerable magnetooptical activity ${ }^{23}$. The periodic corrugation of the DVD grating enables surface plasmon polariton (SPP) resonances at distinct wavelength-angle combinations that are given by

$$
n k_{0}(\omega) \sin \theta_{0}= \pm k_{S P P}(\omega)+\left(\frac{2 \pi}{d}\right) m
$$

where $n$ is the refractive index of the surrounding environment, $k_{0}$ the wave vector of light in free space, $\theta_{0}$ the incidence angle, $d$ the periodicity of the grating and $m$ an integer denoting the order of the SPP. The SPP wave vector is given by $k_{S P P}=k_{0} \sqrt{\varepsilon_{1} \varepsilon_{2} /\left(\varepsilon_{1}+\varepsilon_{2}\right)}$, where $\varepsilon_{1}$ and $\varepsilon_{2}$ are the permittivities of the metallic layer and the surrounding dielectric environment. Due to the thickness of the gold/cobalt multilayer film, we can assume that SPPs are only excited on top of the multilayer film.

\section{PROTOCOL:}

1. Mounting the setup

\subsection{Optics}

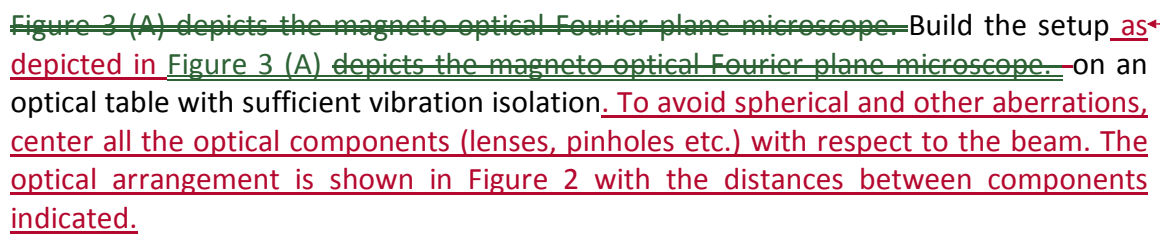
depicted in Figure 3 (A) depicts the magneto-optical Fourier plane microscope. on an optical table with sufficient vibration isolation. To avoid spherical and other aberrations, center all the optical components (lenses, pinholes etc.) with respect to the beam. The optical arrangement is shown in Figure 2 with the distances between components indicated.

Formatted: Indent: Left: $1,27 \mathrm{~cm}$, Space After: $0 \mathrm{pt}$, Line spacing: single, No widow/orphan control 
1.1.1.

1.1.1. Guide the light from the white light source to a monochromator to obtain a monochromatic light beam. See table of materials for details of the setup used in this work. Set the monochromator to a wavelength that has a good intensity and visibility, e.g. $550 \mathrm{~nm}$. A wavelength from the visibile part of the spectrum makes it easier to position the optical elements.

1.1.2. Using a coupling lens, couple the light to a fiber and collimate it with an objective at the fiber termination. Depending on the light source used, this step may be omitted.

1.1.3. Optionally, usePlace a polarizer $250 \mathrm{~mm}$ from the collimating lens to a polarizer tolinearly polarize the beam and directit to a beam splitter 100 $\mathrm{mm}$ from the polarizer to, which guides the light to the microscope objective lens.

1.1.2. NOTE: due to the collimated beam, the indicated positions of the above-4 mentioned components don't affect the optics of the measurements setup and are given just for guidance.

1.1.3 1.1.4. Place the sample on the sample holder equipped with a $x-y-z$ translation stage and a rotation stage that enables a 360-degree sample rotation around the $z-$ axis, i.e the axis of light impinging on the sample.

1.1.4.1.1.5. Mount the objective lens on a translation stage that enables movement in 3 directions. The most crucial of these is the $z$-axis that is needed for focusing onto the sample.

NOTE: The required degrees of freedom for sample and objective lens translation

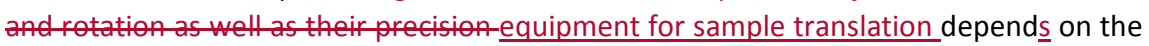
samples used. Large, homogenous samples may be positioned manually while samples with small patterned-useful area will require more careful positioning, especially when using a pinhole to limit the imaged area (see below1.1.7.).

1.1.6. The optics of the beam emerging from the sample is schematically depicted in Figure 2. The infinity corrected objective lens directs wave fronts emerging from each point of the sample into collinear beams. Place a collector lens with $f=200 \mathrm{~mm}$ (tube lens) $330 \mathrm{~mm}$ from the objective to re-focus the beams that are re-focused using a collector lens (tube lens) to form an image at the image plane. Due to the
Formatted: Indent: Left: $1,27 \mathrm{~cm}$, Space After: $0 \mathrm{pt}$, Line spacing: single, No widow/orphan control

Formatted: Indent: Left: $1,27 \mathrm{~cm}$, Space After: $0 \mathrm{pt}$ Line spacing: single, No bullets or numbering, No widow/orphan control

Formatted: Indent: Left: $2,16 \mathrm{~cm}$, No bullets or numbering

Formatted: Highlight 
collinear propagation of the light emanating from the sample, the collector lens can be placed at any distance from the objective lens.

1.1.3. NOTE: As before, the light emerging from the objective lens is collimated. The tube lens should be placed after the beam splitter, however. Using a flip-mirror, the image plane can be alternatively imaged to see the sample in realspace for navigation purposes.

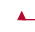

1.1.5 1.1.7. A pinderan place planhole into the image plane a $200 \mathrm{~mm}$ from - on the image planethe collector lens to limit the imaged region to the patterned area. Place the pinhole at the center of the beam. If using ainhole operation, use the real space image of the sample to position it. For samples where the patterned area is larger than the area illuminated by the light beam, this is not necessary.

1.1.6.1.1.8. Place a second lens with $\mathrm{f}=75 \mathrm{~mm}$ (Bertrand lens) $\underline{120 \mathrm{~mm}}$ after the image plane to create a Fourier transform of the angular components of the image. The transform is created at the focus of the second lens and imaged with a sCMOS camera which is placed $75 \mathrm{~mm}$ from the Bertrand lens:

1.1.7.1.1.9. For LMOKE measurements only: Insert an additional polarizer with an angle with respect to the first polarizer between the beam splitter and the collector lens.

\subsection{Magnet}

1.2.1. Connect the magnet to a power supply and mount it so that the magnetic field can be applied on the sample. Choose whether the magnetic field is applied in the longitudinal, transversal or polar direction (Figure 1).

\subsection{Sample preparation}

1.3.1. Dismantle mechanically a commercial DVD disk, subsequently the exposed grating surface can be easily identified due its diffractive proprieties. Use a scotch tape to peel the previously coatings. Having the surface clean, soak it in ethanol for 10 minutes, finally the grating is ready to receive a magneto-plasmonic coating.
Formatted: Highlight

Formatted: Indent: Left: $2,16 \mathrm{~cm}$, No bullets or numbering

Formatted: Highlight 
NOTE: Different commercial optical disks as Blu-ray and CD, may need a different preparation protocol.

1.3.2. Deposit the metal film on the exposed grating by electron-beam evaporation. To ensure small roughness, use evaporation rates smaller than $5 \AA$ /s.

1.3.3. Starting with a $4 \mathrm{~nm} \mathrm{Cr}$ adhesive layer, deposit alternating gold and cobalt layers, finishing with a gold capping layer to ensure protection from oxidation. We used the following number of layers and thicknesses: $\mathrm{Cr}(4 \mathrm{~nm}) / \mathrm{Au}(16 \mathrm{~nm}) /[\mathrm{Co}(14 \mathrm{~nm}) / \mathrm{Au}$ $(16 \mathrm{~nm})] \times 4 / \mathrm{Co}(14 \mathrm{~nm}) / \mathrm{Au}(7 \mathrm{~nm})$.

1.3.4. Perform optical or electron microscopy (Figure 4(A)) to verify the sample surface conditions, in case of homogeneity and low defects proceed with the measurement.

\section{Measurement procedure}

z.

1.4.2.1. Sample positioning

1.4.1.2.1.1. As an illustrative sample, we will measure a DVD grating covered with magnetoplasmonic $\mathrm{Au} / \mathrm{Co} / \mathrm{Au}$ film. Due to the periodic corrugation of the grating, SPPs can be excited at certain angles of incidence based on the wavefront wavelength.

2.1.2. Mount the sample on the sample holder using a small drop of silver paint. Let the silver paint dry for 10 minutes.

1.4.2.2.1.3. Insert a flip mirror after the image plane to enable real space imaging of the sample. Insert a lens L1 with $\mathrm{f}=125 \mathrm{~mm}$ so that the image plane is in focus and $\underline{L} 2$ with $\mathrm{f}=250 \mathrm{~mm}$ to $135 \mathrm{~mm}$ distance from L1. Finally place a CCD camera $210 \mathrm{~mm}$ from $L 2$ to capture a magnified image of the image plane. Move lenses $L 1$ and $L 2$ until you see the pinhole placed in image plane in good focus in the CCD camera. Flip the flip mirror so that the real space image of the sample can be seen.

1.4.3-2.1.4. Move the objective lens towards the sample untilyou see the sample in good focus in the CCD camera. it is in good focus.
Formatted: Indent: Left: $0,63 \mathrm{~cm}$, No bullets or numbering

Formatted: Outline numbered + Level: $3+$ Numbering Style: $1,2,3, \ldots+$ Start at: 1 + Alignment: Left + Aligned at: $1,27 \mathrm{~cm}+$ Indent at: $2,16 \mathrm{~cm}$ 


\subsubsection{Optical reflectivity measurement}

1.5.1.2.2.1. Using the real space image of the sample, position the light spot over a reflective (unpatterned) part of the sample. In our DVD-grating we use the continuous metallic film at edge of the DVD disk. Flip the flip mirror so that you can see the BFP of the microscope.

1.5.2.2.2.2. Select the area of the back focal planespot that corresponds to the desired polarization state. The relationship between polarization and position in the back focal plane is shown in figure 3 (B). Select an when the objective focuses an incoming linearly polarized beam onto the sample, both TE-and TM-polarized light is present, depending on the incidence angle of the beam. Thus, the area of interest (AOI) $\mathrm{Ean}$ be selected to be aas a rectangular cross section of the objective back focal plane (blue rectangle in Figure 3 (C)) along the axis that corresponds to TMp-polarization. In the Labview software used in this manuscript, this is achieved by selecting the AOI using the cursor selectors. The software then a-As long as the rectangular cross section selected is narrow enough, tverages thehe intensities along the shortits $\forall$ dimension of the rectangle and treatsean be averaged and the resulting spectrum treated as a 1D array of data where eaç emission angle of the sample.

NOTE: In plasmonic gratings, only TM-polarized light, i.e. EM radiation with electric field perpendicular to the grating grooves, can excite the plasmon resonances. Thus, depending on the grating orientation, it is necessary to select the correct polarization state by either choosing vertical or horizontal slice of the BFP.

1.5.3.2.2.3. Measure the spectrum of the light source by clicking "measure normalization spectrum", which will be used later to normalize the measured reflectivity data. As each wavelength yields a $1 \mathrm{D}$ set of data points, the full spectrum of the light source is saved as aa 2D tensor where each data point represents a combination of wavelength and angle.

1.5.4.2.2.4. Using again the real space image of the sample, position the light source over the photonic crystal of interest. When you switch back to BFP,,$¥$ ₹ou should be able to see the plasmon modes as dark lines crossing the back focal plane. The lines move as the wavelength of the incident light is modified.
Formatted: Not Highlight

Formatted: Not Highlight

Formatted: Not Highlight

Formatted: Not Highlight

Formatted: Not Highlight

Formatted: Not Highlight

Formatted: Not Highlight

Formatted: Not Highlight 
1.5.5-2.2.5. Using the same $\mathrm{AOI}$ and averaging proceduremeasurement settings (i.e. exposure times, number of averages), measure the reflection spectrum of the photonic crystal by clicking "measure reflection spectrum".

1.5.6.2.2.6. To account for the spectral variation in light source intensity, normalize the obtained spectrum by the spectrum of the light source. This will yield a 2D array of numbers from 0 to 1 where 1 corresponds to fully reflective and 0 to fully absorptive conditions.

\subsection{Magneto-optical measurement}

2.3.1. Start the magneto-optical measurement by measuring a hysteresis loop using an angle and wavelength that are known to correspond to a good magneto-optical response, usually these conditions can be found close to the SPP excitations. To do that, choose a small AOI near the SPP excitations and measure single loop.

NOTE: The data analysis needed to quantify the magneto-optical activity depends on the type of magnetism that the sample exhibits. Here, we assume a ferromagnetic response and treat the results accordingly. Dia- or paramagnetic response is essentially linear to applied magnetic field and can be quantified as change in optical properties per applied magnetic field unit. Ferromagnetic materials exhibit a non-linear permittivity that requires additional consideration when defining the magneto-optical response (See Figure $3(D)$ ). The TMOKE is defined as change in reflected intensity as the function of applied magnetic field, i.e. TMOKE = $I(+M)-I(-M) / I(M=0)$, where $I(M)$ is the intensity reflected by the sample at magnetization state $M$.

2.3.2. Using the hysteresis loop measured in 2.3.1., choose the range of magnetic fields to loop. For ferromagnetic samples, loop the fields from a fully saturated state to an oppositely saturated state, extending the range comfortably over the saturation field. Later, use the points measured in the saturated state to analyze and remove any dia- or paramagnetic contributions that can be verified by their linear contribution.
Formatted: Not Highlight

Formatted: Not Highlight

Formatted: Indent: Left: $1,4 \mathrm{~cm}$, No bullets or numbering

Formatted: Not Highlight

Formatted: Font:

Formatted: Indent: Left: $2,16 \mathrm{~cm}$, No bullets or numbering

Formatted: Font:

Formatted: Normal, No bullets or numbering

Formatted: Font: Not Highlight

Formatted: Indent: Left: $2,16 \mathrm{~cm}$, No bullets or numbering 
-Finally, measure the intensity reflected by the sample at each magnetic field point defined, repeating over multiple loops if desired. Each wavelength and magnetization point -yields a single 1D array of numerical data (i.e. measured light intensity) where each point of the array corresponds to a particular angle. 2.2

2.2.1. The TMOKE is defined as change in reflected intensity as the function of applied magnetic field, i.e. TMOKE $=I(+M)-I(-M) / I(M=0)$, where $/(M)$ is the intensity reflected by the sample at magnetization state $\mathrm{M}$.

2.3.3.

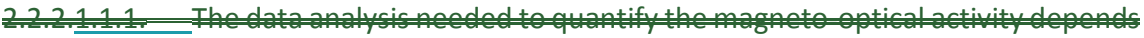
on the ty of manetism that the sampexhits. Here, we asume-a

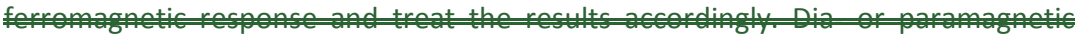

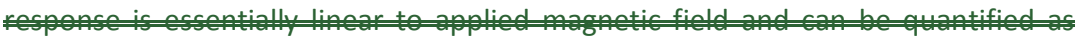

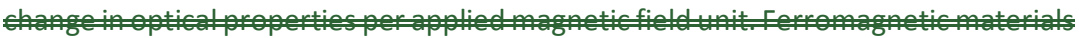

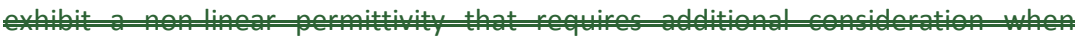
.

2.2.3. In order to stablish the field needed to saturate the magnetization, it is usefulto measure the hysteresis loop of the sample using an angle and wavelength that are known to correspond to a good magneto-optical response. Additionally, this is a good practice for verifying that the magneto-optical measurement is correctly set up and doesn't include any spurious signals that result from aberrations such as sample movements due to applied magnetic fields. A single hysteresis loopis enough to find out the saturation magnetization, as the magnetic properties of the sample don't depend on the direction and wavelength of light incident onto it. However, the shape of the hysteresis loop can be affected in some cases, due to the fact that different wavelengths, polarizations and incidence angles may probe different magnetization components.

2.2.4. Using the hysteresis loops measured before, choose the range of magnetic fields toloop. It is recommended to loop the fields from a fully saturated state to an oppositely saturated state, extending the range comfortably over the saturation field needed. Later, the points measured in the saturated state can be used to analyse and remove any dia-or paramagnetic contributions that can be verified by their linear contribution. On the other hand, the range should not be overly extended to avoid any mechanical movements that can be caused by applying strong magnetic fields. For ferromagnetic samples, the measurement points in the chosen magnetic field range can be selected so that fewer measurements are made in the intermediate range and most measurements are carried in the wo saturated states as the magneto-optical information will be later extracted from this data.

Page 8 of 6

revised November 2018
Formatted: Outline numbered + Level: $3+$ Numbering Style: $1,2,3, \ldots+$ Start at: $1+$ Alignment: Left + Aligned at: $1,27 \mathrm{~cm}+$ Indent at: $2,16 \mathrm{~cm}$

Formatted: List Paragraph, Space After: 0 pt, Line spacing: single, Outline numbered + Level: $1+$ Numbering Style: $1,2,3, \ldots+$ Start at: $1+$ Alignment: Left + Aligned at: $0 \mathrm{~cm}+$ Indent at: $0,63 \mathrm{~cm}$

Formatted: Indent: Left: $0,63 \mathrm{~cm}$, Space After: $0 \mathrm{pt}$, Line spacing: single, Outline numbered + Level: $1+$

Numbering Style: $1,2,3, \ldots+$ Start at: $1+$ Alignment: Left + Aligned at: $0 \mathrm{~cm}+$ Indent at: $0,63 \mathrm{~cm}$, No widow/orphan control

Formatted: Outline numbered + Level: $3+$ Numbering Style: $1,2,3, \ldots+$ Start at: $1+$ Alignment: Left + Aligned at: $1,27 \mathrm{~cm}+$ Indent at: $2,16 \mathrm{~cm}$

Formatted: Space After: 0 pt, Line spacing: single, No widow/orphan control

Formatted: Normal, Space After: 0 pt, Line spacing: single, No bullets or numbering 
NOTE: If the intensity level of the source is not stable over time, the magnetic field should be tooped quickly to avoid the power drift affecting the measured hysteresis loops. Typically, source power levels drift more slowly than a hysteresis loop can be measured, making it possible to measure the TAMOKE contrast even under these conditions. If the signal is noisy and more averaging is needed, the averaging can be realized by increasing the amount of loops measured Father than the number of frames at each magnetic field point.

NOTE: While ferromagnetic materials in general maintain their magnetization state, the magnetic field should be applied in situ. Due to the small magnitude of the magneto-optical effects, removing the sample for magnetization reversal is usually not recommended due to the difficulty of re-inserting the sample into exactly the same position as it was before the magnetization reversal.

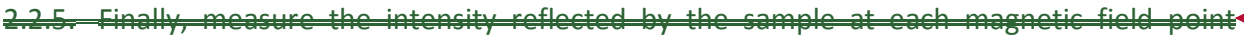

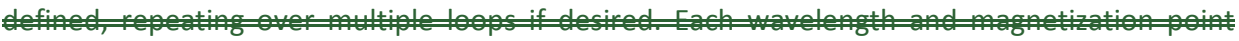

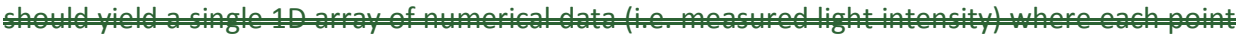
of

\section{Data analysis}

3.1. Using the hysteresis loop of the sample measured at step 2.3.1, assign each frame measured at step 2.3.3. to either of the saturated states or to the intermediate state (Figure $3(\mathrm{C})$ ).

3.2. Discard the intermediate frames and calculate the magneto-optical activity can becalculated from the measured intensities by TMOKE = $2(I(+M)-I(-M)) /(I(+M)+I(-M))$, where the operations are carried out separately for each angular and wavelength data point. As TMOKE is expressed as a relative intensity change, the results don't have to be normalized to the lamp spectrum.

3.3. If the sample presents large paramagnetic (or more rarely, diamagnetic) activity that needs to be subtracted for a reliable comparison between the saturated magnetic states, subtract the linear contribution arising from para- or diamagnetic activity by fitting a line (again, pixelwise separately for each angle and wavelength point) on the points at measured at saturation and remove the linear contribution.

$$
\text { 3. }
$$

3.1. The origin of the magneto-optical effect is the interaction of light between the magnetization of the optical crystal. In dia and paramagnetic materials the magnetization and applied external magnetic fields have a linear relationship and thus

Page 9 of 6
Formatted: Space After: $0 \mathrm{pt}$, Line spacing: single, No widow/orphan control

Formatted: Indent: Left: $0 \mathrm{~cm}$

Formatted: Indent: Left: $0 \mathrm{~cm}$

Formatted: Normal, Space After: 0 pt, Line spacing: single, No bullets or numbering

Formatted: Normal, Indent: Left: $0 \mathrm{~cm}$, Space After: 0 pt, Line spacing: single

Formatted: Indent: Left: $0,63 \mathrm{~cm}$, No bullets or numbering

Formatted: Indent: Left: $1,4 \mathrm{~cm}$, No bullets or numbering

Formatted: Outline numbered + Level: 2 + Numbering Style: $1,2,3, \ldots+$ Start at: $1+$ Alignment: Left + Aligned at: $0,63 \mathrm{~cm}+$ Indent at: $1,4 \mathrm{~cm}$

Formatted: Indent: Left: $1,4 \mathrm{~cm}$, No bullets or numbering

Formatted: Indent: Left: $1,27 \mathrm{~cm}$, Space After: $0 \mathrm{pt}$ Line spacing: single, No widow/orphan control

Formatted: Indent: Left: $1,27 \mathrm{~cm}$, Space After: $0 \mathrm{pt}$, Line spacing: single, No bullets or numbering, No widow/orphan control

Formatted: Indent: Left: $1,27 \mathrm{~cm}$, Space After: $0 \mathrm{pt}$, Line spacing: single, No widow/orphan control 
the magneto-optical activity can be measured as quantity that depends on external field, for example in the case of TMOKE its unit can be given as $\triangle I / T$, i.e. change in intensity per Testa.

3.2. This is not the case of ferromagnetic materials, where the relationship between the applied field and the magnetization is decisively non linear. The defining feature of ferromagnetic materials is their spontaneous magnetization, that exists also in the absence of external magnetic fields. A sufficiently powerful external magnetic field can align the spontaneous magnetic moments of the material, resulting in a saturated magnetization state. Comparing the magneto-optical activity between two saturated magnetization-states of opposing magnetization direction is therefore a good wy to quantify the magneto-optical activity of ferromagnetic materials and nanostructures that incorporate them.

3.3. Typical response of a ferromagnetic material to an external magnetic field is shown in Figure 3 (D). 4 special magnetization states can be distinguished: two opposite saturated states where the magnetic moments are almost fully aligned due to the effect of the external magnetic field (blue rectangles) and two opposing remanent magnetization states where the spontaneous magnetization of the sample persists in the absence of external magnetic field (red circles).

3.4. Previously in step 2.3., the hysteresis loop of the sample under study was measured tofind out the magnetic field needed to saturate it. Looking at the frames measured in 2.4., each one of them can be assigned to either of the saturated states or to the intermediate state. The intermediate frames are discarded and the magneto-optical activity can becalculated from the measured intensities by TMOKE $=2(I(+M)-I(-M)) /(I(+M)+I(-M))$, the operations are carried out separately for each angular and wavelength data point. As TMOKE is expressed as a relative intensity change, the results don't have to be normalized to the lamp spectrum.

3.4.1. Sometimes, the sample presents large paramagnetic (or more rarely, diamagnetic)activity that needs to be subtracted for a reliable comparison between the saturated magnetic states. The linear contribution arising from para-or diamagnetic activity to the magneto-optical signal can be subtracted by fitting a line lagain, pixelwise separately for each angle and wavelength point) on the points at saturation and remove the linear contribution.

Page 10 of 6

revised November 2018
Formatted: Normal, No bullets or numbering

Formatted: Normal, No bullets or numbering

Formatted: Normal, Space After: 8 pt, Line spacing: Multiple 1,08 li 


\section{REPRESENTATIVE RESULTS:}

Figure 4 (A) shows a SEM micrograph of a commercial DVD grating covered $\mathrm{Au} / \mathrm{Co} / \mathrm{Au}$ multilayer that was used a demonstration sample in our experiments. Its optical and magneto-optical spectra are shown in Figure 4 (B) and (C), respectively. Details on sample fabrication are presented in ${ }^{23}$ Black lines 4 (A) an (B) show the plasmon dispersion relations calculated from equation 1. The permittivity of the $\mathrm{Au} / \mathrm{Co} / \mathrm{Au}$ multilayer is taken from supplementary Data File 1 $i^{24}$ where a similar multilayer was measured using spectroscopic ellipsometry. Periodicity of the grating is assumed to be $740 \mathrm{~nm}$. The calculated dispersion lines correspond to a conspicuous dip in reflectivity in 4 (A) that result from the incident radiation being converted into SPPs and dissipated via ohmic damping.

The relationship between the pixel positions in the back focal plane (Figure $3(C)$ ) and angle of emission can be established as follows: the maximum angle $\theta_{\max }$ at which the objective can accept light is given by formula $N A=n \sin \theta_{\max }$ and depends on the numerical aperture $N A=$ 0.8 and refractive index of the surrounding medium (air, $n=1$ ). This is the angle that corresponds to the extremes of the illuminated area of the Fourier plane. The pixels between them can be assigned a number in a linear manner from $-N A$ to $+N A$ that reflects the numerical aperture at their position and their corresponding angle is then given by the inverse sine of this number (divided by $n$ if necessary).

Figure 4 (C) depicts the magneto-optical spectrum of the plasmonic grating. Here, the plasmon lines are accompanied by an increase in magneto-optical activity that abruptly reverses at the SPP. The lineshape can be explained by the fact that the magnetization slightly changes the SPP excitation conditions, thus resulting in two different SPPs for opposite magnetization states. When the reflectivities of the two slightly displaced states are subtracted from each other, a characteristic derivative lineshape is obtained ${ }^{15-17}$. The plasmon linewidths of the plasmon resonances as well as the resulting magneto-optical spectra depend strongly on the material parameters of the metal multilayer ${ }^{25,26}$.

We note that due to the geometry of the grating, the magnetic easy axis is oriented along the grating itself and very large magnetic fields are needed to saturate it out of this plane, for this reason LMOKE measurements with this particular sample are not feasible.-

\section{FIGURE AND TABLE LEGENDS:}

Figure 1: Different geometries where magneto-optical effects can be observed. Polar (A), Longitudinal (B) and Transverse (C) Magneto-optical Kerr effects are observed in reflection while Faraday (D) and Voigt (E) effects occur in transmission through magnetized medium.

Figure 2: $(A)$ : Schematic depiction of light propagation in the Fourier plane microscopic setup. The distinct angular components (depicted with red, black and blue rays) are spatially separated at the back focal plane. (B): Schematic depiction of the light propagation in the real space microscope. Lenses L1 and L2 form a telescope that images the at the image plane to the camera. The distances between the components on the optical table are highlighted below each setup. 
Red numbers indicate that the distance is critical for image formation.

Figure $3(A)$ : Components of the Fourier space microscope (B): Schematic depiction of the polarization states of the light focused by the objective. Incident linearly (along $\mathrm{x}$-direction) polarized light impinges on the sample as both TE $\mathrm{p}$ - and TMs-polarized depending on the part of the objective where the ray originates. $(C)$ Intensity at the back focal plane of the microscope at $\lambda=600 \mathrm{~nm}$ when measuring the DVD grating. The black absorptive lines indicate SPP resonances that are also visible in Figure 4 (B) and (C). AOI can be chosen as the blue rectangle to measure response to TMp-polarized light or red for TEs-polarized. (D) Schematic hysteresis loop of a ferromagnetic material demonstrating the typical nonlinear response to applied magnetic fields.

Figure 4: (A): SEM micrograph of a commercial DVD grating covered with $\mathrm{Au} / \mathrm{Co} / \mathrm{Au}$ multilayer. Angular resolved reflectivity $(B)$ and magneto-optical activity $(C)$ map of the DVD grating with periodicity of $740 \mathrm{~nm}$.

\section{DISCUSSION:}

We have introduced a measurement setup and protocol to obtain angular resolved magnetooptical spectra of optical crystals. In particular, the case of ferromagnetic materials, that requires additional data analysis to account for the nonlinear permeability of the material, has been laid out. Angular resolved magneto-optical spectroscopy presents an additional advantage over nonangular resolved methods that the confined modes can be more readily identified as they appear as clearly defined bands in both optical and magneto-optical spectra. The approach we show here can be readily adapted to various kinds of photonic crystals and is not limited to surface plasmon resonances.

Most common modification to the technique would be its adaptation to measure longitudinal and/or polar Kerr effects, that manifest as polarization rotation rather than intensity effects. To measure polarization rotation, additional polarizer must be placed between the beam splitter and the collector lens to make the intensity detected at the camera proportional to polarization rotation. This polarizer should be placed at 45 angle with the polarization of the light incident on the sample to maximize the magneto-optical signal ${ }^{27}$.

Common pitfalls in the measurement technique include incorrect mounting of sample so that it can move when a magnetic field is applied. This can be aggravated by using magnetic metal such as iron in the sample holder. Even small quantities of magnetic metals such as small screws can result in movements that mask the magneto-optical effect entirely. A moving sample results typically in a "banana-like" incorrect hysteresis loop. Therefore, proper care needs to be taken in mounting the sample and making sure it is firmly in place before measurements. To confirm proper mounting of the sample, it is recommended measure hysteresis loops using a wavelength/angle combination that is known to result in good signal and to confirm that its shape is as expected and that any artefacts from sample movement or other aberrations is not present.

As the measurement of the hysteresis loop requires looping over a range of applied magnetic field, the measurement takes some time. If the intensity level of the source is not stable over

Page 12 of 6

revised November 2018 
time, the magnetic field should be looped quickly to avoid the power drift affecting the measured hysteresis loops. Typically, source power levels drift more slowly than a hysteresis loop can be measured, making it possible to measure the TMOKE contrast even under these conditions. If the signal is noisy and more averaging is needed, the averaging can be realized by increasing the amount of loops measured rather than the number of frames at each magnetic field point.

This technique relies on applying the magnetic field in-situ. While ferromagnetic materials in usually maintain their magnetization state in the absence of applied magnetic fields, due to the small magnitude of the magneto-optical effects, removing the sample for manipulating the magnetization results in failure due to the difficulty of re-inserting the sample into exactly the same position as it was before the magnetization reversal.

The method that we have presented here relies on sensitive detection equipment and stable light sources. In standard mAMagneto-optical Kerr spectrometry in longitudinal or polar Kerr configuration, a photoelastic modulator is often used to enhance the signal-to-noise ratio and to separate rotation and ellipticity components from each other ${ }^{27,28}$. However, the modulation frequency of a photoelastic modulator is typically more than $50 \mathrm{kHz}$ which makes it very difficult to use with a microscope camera. Therefore, to obtain the best possible signal-to-noise ratio for a Fourier space magneto-optical microscope, it is necessary to invest in cameras and light sources with good stability.

In longitudinal and polar magneto-optical measurements, the intensity of light incident on the camera is greatly reduced due to the crossed polarizer placed before it, which puts additional requirements on the camera equipment needed for to detect the much weaker signal.

\section{ACKNOWLEDGMENTS:}

We acknowledge financial support by the Spanish Ministerio de Economía y Competitividad through projects MAT2017-85232-R (AEI/FEDER,UE), Severo, Ochoa (SEV-2015-0496) and by the Generalitat de Catalunya (2017, SGR 1377), by CNPq - Brazil, and by the European Comission (Marie Skłodowska-Curie IF EMPHASIS - DLV-748429).

\section{DISCLOSURES:}

The authors have nothing to disclose.

\section{REFERENCES:}

1. Bayer, M. et al. Optical Modes in Photonic Molecules. Physical Review Letters. 81 (12), 25822585, doi: 10.1103/PhysRevLett.81.2582 (1998).

2. Blanco, A. et al. Large-scale synthesis of a silicon photonic crystal with a complete threedimensional bandgap near 1.5 micrometres. Nature. 405 (6785), 437, doi: 10.1038/35013024 (2000).

Formatted: Bibliography, Adjust space between Latin and Asian text, Adjust space between Asian text and numbers 
3. Rybin, M.V. et al. High-Q Supercavity Modes in Subwavelength Dielectric Resonators. Physical Review Letters. 119 (24), 243901, doi: 10.1103/PhysRevLett.119.243901 (2017).

4. Joannopoulos, J.D., Villeneuve, P.R., Fan, S. Photonic crystals. Solid State Communications. 102 (2), 165-173, doi: 10.1016/S0038-1098(96)00716-8 (1997).

5. Englund, D., Fushman, I., Vuckovic, J. General recipe for designing photonic crystal cavities. Optics Express. 13 (16), 5961-5975, doi: 10.1364/OPEX.13.005961 (2005).

6. Yablonovitch, E. Inhibited Spontaneous Emission in Solid-State Physics and Electronics. Physical Review Letters. 58 (20), 2059-2062, doi: 10.1103/PhysRevLett.58.2059 (1987).

7. Yablonovitch, E. Photonic band-gap structures. JOSA B. 10 (2), 283-295, doi: 10.1364/JOSAB.10.000283 (1993).

8. Noda, S., Tomoda, K., Yamamoto, N., Chutinan, A. Full Three-Dimensional Photonic Bandgap Crystals at Near-Infrared Wavelengths. Science. 289 (5479), 604-606, doi: 10.1126/science.289.5479.604 (2000).

9. John, S. Strong localization of photons in certain disordered dielectric superlattices. Physical Review Letters. 58 (23), 2486-2489, doi: 10.1103/PhysRevLett.58.2486 (1987).

10. Krauss, T.F. Slow light in photonic crystal waveguides. Journal of Physics D: Applied Physics. 40 (9), 2666-2670, doi: 10.1088/0022-3727/40/9/S07 (2007).

11. Huang, X., Lai, Y., Hang, Z.H., Zheng, H., Chan, C.T. Dirac cones induced by accidental degeneracy in photonic crystals and zero-refractive-index materials. Nature Materials. 10 (8), 582-586, doi: $10.1038 /$ nmat3030 (2011).

12. Wagner, R., Heerklotz, L., Kortenbruck, N., Cichos, F. Back focal plane imaging spectroscopy of photonic crystals. Applied Physics Letters. 101 (8), 081904, doi: 10.1063/1.4746251 (2012).

13. Zhang, D. et al. Back focal plane imaging of directional emission from dye molecules coupled to one-dimensional photonic crystals. Nanotechnology. 25 (14), 145202, doi: 10.1088/0957-4484/25/14/145202 (2014).

14. Vasista, A.B., Sharma, D.K., Kumar, G.V.P. Fourier Plane Optical Microscopy and Spectroscopy. Digital Encyclopedia of Applied Physics. 1-14, doi: 10.1002/3527600434.eap817 (2019).

15. Belotelov, V.I., Doskolovich, L.L., Zvezdin, A.K. Extraordinary Magneto-Optical Effects and Transmission through Metal-Dielectric Plasmonic Systems. Physical Review Letters. 98 (7), 077401, doi: 10.1103/PhysRevLett.98.077401 (2007).

16. Belotelov, V.I. et al. Enhanced magneto-optical effects in magnetoplasmonic crystals. Nature Nanotechnology. 6 (6), 370, doi: 10.1038/nnano.2011.54 (2011).

17. Chetvertukhin, A.V. et al. Magneto-optical Kerr effect enhancement at the Wood's anomaly in magnetoplasmonic crystals. Journal of Magnetism and Magnetic Materials. 324 (21), 3516-3518, doi: 10.1016/i.jmmm.2012.02.079 (2012).

18. Kataja, M., Hakala, T.K., Julku, A., Huttunen, M.J., van Dijken, S., Törmä, P. Surface lattice resonances and magneto-optical response in magnetic nanoparticle arrays. Nature Communications. 6, 7072, doi: 10.1038/ncomms8072 (2015).

19. Kataja, M. et al. Hybrid plasmonic lattices with tunable magneto-optical activity. Optics Express. 24 (4), 3652-3662, doi: 10.1364/OE.24.003652 (2016).

20. Kalish, A.N. et al. Magnetoplasmonic quasicrystals: an approach for multiband magnetooptical response. Optica. 5 (5), 617-623, doi: 10.1364/OPTICA.5.000617 (2018).

Page 14 of 6

revised November 2018 
21. Borovkova, O.V. et al. TMOKE as efficient tool for the magneto-optic analysis of ultra-thin magnetic films. Applied Physics Letters. 112 (6), 063101, doi: 10.1063/1.5012873 (2018).

22. Kurvits, J.A., Jiang, M., Zia, R. Comparative analysis of imaging configurations and objectives for Fourier microscopy. JOSA A. 32 (11), 2082-2092, doi: 10.1364/JOSAA.32.002082 (2015).

23. Cichelero, R., Oskuei, M.A., Kataja, M., Hamidi, S.M., Herranz, G. Unexpected large transverse magneto-optic Kerr effect at quasi-normal incidence in magnetoplasmonic crystals. Journal of Magnetism and Magnetic Materials. 476, 54-58, doi: 10.1016/i.jmmm.2018.12.036 (2019).

24. Cichelero, R., Kataja, M., Campoy-Quiles, M., Herranz, G. Non-reciprocal diffraction in magnetoplasmonic gratings. Optics Express. $26 \quad(26), 34842-34852$, doi: 10.1364/OE.26.034842 (2018).

25. Melo, L.G.C., Santos, A.D., Alvarez-Prado, L.M., Souche, Y. Optimization of the TMOKE response using the ATR configuration. Journal of Magnetism and Magnetic Materials. $310(2$, Part 3), e947-e949, doi: 10.1016/i.jmmm.2006.10.998 (2007).

26. Regatos, D., Sepúlveda, B., Fariña, D., Carrascosa, L.G., Lechuga, L.M. Suitable combination of noble/ferromagnetic metal multilayers for enhanced magneto-plasmonic biosensing. Optics Express. 19 (9), 8336-8346, doi: 10.1364/OE.19.008336 (2011).

27. Polisetty, S. et al. Optimization of magneto-optical Kerr setup: Analyzing experimental assemblies using Jones matrix formalism. Review of Scientific Instruments. 79 (5), 055107, doi: $10.1063 / 1.2932445$ (2008).

28. Sato, K. Measurement of Magneto-Optical Kerr Effect Using Piezo-Birefringent Modulator. Japanese Journal of Applied Physics. 20 (12), 2403, doi: 10.1143/JJAP.20.2403 (1981).

1. Bayer, M. et al. Optical Modes in Photonic Molecules. Physical Review Letters.81 (12), 2582= 2585, doi: 10.1103/PhysRevLett.81.2582 (1998).

Z. Blanco, $A$. et al. Large-scale-synthesis of a silicon photonic crystal with a complete threedimensional bandgap near 1.5 micrometres. Nature. 405 (6785), 437, doi: 10.1038/35013024 (2000).

3. Rybin, M.V. et al. High-\$Q\$S Supercavity Modes in Subwavelength Dielectric Resonators. Physical Review Letters. 119 (24), 243901, doi: 10.1103/PhysRevlett.119.243901 (2017).

4. Joannopoulos, J.D., Villeneuve, P.R., Fan, S. Photonic crystals. Solid State Communications. 102 (2), 165-173, doi: 10.1016/s0038-1098(96)00716-8(1997).

5. Englund, D., Fushman, 1., Vuckovic, J. General recipe for designing photonic crystal cavities. Optics Express. 13 (16), 5961-5975, doi: 10.1364/OPEX.13.005961 (2005).

6. Yablonovitch, E. Inhibited Spontaneous Emission in Solid-State Physics and Electronics. Physical Review Letters. 58 (20), 2059-2062, doi: 10.1103/PhysRevLett.58.2059 (1987).

7. Yablonovitch, E. Photonic band-gap structures. JOSA B. 10 (2), 283-295, doi: 10.1364/JOSAB.10.000283 (1993).

8. Noda, S., Tomoda, K., Yamamoto, N., Chutinan, A. Full Three-Dimensional Photonic Bandgap Crystals at Near-Infrared Wavelengths. Science. 289 (5479), 604-606, doi: 10.1126/science.289.5479.604 (2000).

9. John, S. Strong localization of photons in certain disordered dielectric superlattices. Physical Review Letters. 58 (23), 2486-2489, doi: 10.1103/PhysRevLett.58.2486 (1987).

Page 15 of 6

revised November 2018 
10. Krauss, T.F. Slow light in photonic crystal waveguides. Journal of Physics D: Applied Physics. 40 (9), 2666-2670, doi: 10.1088/0022-3727/40/9/507 (2007).

11. Huang, $X .$, tai, Y., Hang, Z.H., Zheng, H., Chan, C.T. Dirac cones induced by accidental degeneracy in photonic crystals and zero-refractive-index materials. Nature Materials. 10 (8), 582-586, doi: 10.1038/mmat3030(2011).

12. Wagner, R., Heerklotz, L., Kortenbruck, N., Cichos, F. Back focal plane imaging spectroscopy of photonic crystals. Applied Physics Letters. 101 (8), 081904, doi: $10.1063 / 1.4746251(2012)$.

13. Zhang, D. et al. Back focal plane imaging of directional emission from dye molecules coupled to one-dimensional photonic crystals. Nanotechnology. 25 (14), 145202, doi: $10.1088 / 0957-4484 / 25 / 14 / 145202$ (2014).

14. Vasista, A.B., Sharma, D.K., Kumar, G.V.P. Fourier Plane Optical Microscopy and Spectroscopy. digital Encyclopedia of Applied Physics. 1-14, doi: 10.1002/3527600434.eap817 (2019).

15. Belotelov, V.I., Doskolovich, L.L., Zvezdin, A.K. Extraordinary Magneto-Optical Effects and Transmission through Metal-Dielectric Plasmonic Systems. Physical Review Letters. 98 (7), 077401, doi: 10.1103/PhysRevett.98.077401 (2007).

16. Belotelov, V.I. et al. Enhanced magneto-optical effects in magnetoplasmonic crystals. Aature Nanotegy. 6(6), 370, doi: 10.1038/mnano.2011.54(2011).

17. Chetvertukhin, A.V. et al. Magneto-optical Kerr effect enhancement at the Wood's anomaly in magnetoplasmonic crystals. Journal of Magnetism and Magnetic Materials. 324 (21), 3516-3518, doi: 10.1016/j.jmmm.2012.02.079 (2012).

18. Kataja, M., Hakala, T.K., Julku, A., Huttunen, M.J., van Dijken, S., Törmä, P. Surface lattice resonances and magneto-optical response in magnetic nanoparticle arrays. Nature Communications. 6, 7072, doi: 10.1038/ncomms8072 (2015).

19. Kataja, M. et al. Hybrid plasmonic lattices with tunable magneto-optical activity. Optics Express. 24 (4), 3652-3662, doi: 10.1364/0E.24.003652 (2016).

20. Kalish, A.N. et al. Magnetoplasmonic quasicrystals: an approach for multiband magnetooptical response. Optica. 5 (5), 617-623, doi: 10.1364/OPTICA.5.000617 (2018).

21. Borovkova, O.V. et al. TMOKE as efficient tool for the magneto-optic analysis of ultra-thin magnetic films. Applied Physics tetters. 112 (6), 063101, doi: 10.1063/1.5012873 (2018).

22. Kurvits, J.A., Jiang, M., Zia, R. Comparative analysis of imaging configurations and objectives for Fourier microscopy. JOSA A. 32 (11), 2082-2092, doi: 10.1364/JOSAA.32.002082 (2015).

23. Cichelero, R., Oskuei, M.A., Kataja, M., Hamidi, S.M., Herranz, G. Unexpected large transverse magneto-optic Kerr effect at quasi-normal incidence in magnetoplasmonic crystals. tournal of Magnetism and Magnetic Materials. 476, 54-58, doi: 10.1016/j.jmmm.2018.12.036 (2019).

24. Cichelero, R., Kataja, M., Campoy-Quiles, M., Herranz, G. Non-reciprocal diffraction in magnetoplasmonic gratings. Optics Express. 26 (26), 34842-34852, doi: 10.1364/OE.26.034842 (2018).

25. Melo, L.G.C., Santos, A.D., Alvarez-Prado, L.M., Souche, Y. Optimization of the TMOKE response using the ATR configuration. Journal of Aagnetism and Magnetic Materials. 310 (2, Part 3), e947-e949, doi: 10.1016/j.jmmm.2006.10.998 (2007).

Page 16 of 6

revised November 2018 
26. Regatos, D., Sepúlveda, B., Fariña, D., Carrascosa, L.G., Lechuga, L.M. Suitable combination of noble/ferromagnetic metal multilayers for enhanced magneto-plasmonic biosensing. Opties Express. 19 (9), 8336-8346, doi: 10.1364/0E.19.008336 (2011).

27. Sato, K. Measurement of Magneto-Optical Kerr Effect Using Piezo-Birefringent Aodulator. Japanese Journal of Applied Physies. 20 (12), 2403, doi: 10.1143/fyAP.20.2403 (1981).

28. Polisetty, S. et al. Optimization of magneto-optical Kerr setup: Analyzing experimental assemblies using Jones matrix formalism. Review of Scientific Instruments. 79 (5), 055107, doi: $10.1063 / 1.2932445$ (2008). 